\title{
El Territorio en disputa: estrategias políticas y movimientos socioterritoriales
}

(4) Sam Halvorsen

School of Geography. Queen Mary University of London. Reino Unido.

Recibido: 4 de noviembre de 2019. Aceptado: 9 de abril de 2020.

\section{Resumen}

El territorio ha sido foco de análisis en diversas investigaciones. En gran parte de la literatura anglosajona ha sido conceptualizado como un resultado desde arriba de control del espacio, frecuentemente encarnado por el Estado. En contraste, los trabajos latinoamericanos suelen hacer hincapié en la potencia emancipadora de las luchas por el territorio en búsqueda de la autonomía. Este artículo avanza en una comprensión del concepto de territorio como estrategias superpuestas e intrincadas y, de esa manera, va más allá de los intentos por entenderlo como una dicotomía involucrada en procesos de construcción desde arriba (el Estado, por ejemplo) o desde abajo (los movimientos sociales). Para ello, examina literatura reciente sobre movimientos socioterritoriales -movimientos para los cuales la apropiación del espacio es el objetivo central de su estrategia- y explora algunas relaciones de poder. El artículo está organizado en tres secciones, las cuales corresponden a estrategias particularmente visibles de los movimientos socioterritoriales en las últimas décadas en la región.

PALABRAS CLAVE: TERRITORIO. ESTRATEGIA. MOVIMIENTOS SOCIOTERRITORIALES RELACIONES DE PODER. AMÉRICA LATINA.

\section{Contested territory: political strategies and socioterritorial movements}

\begin{abstract}
Territory has been focus of analysis in diverse literatures. In much anglophone literature it has been conceptualized as a top-down outcome of controlling space, usually by the state. Latin American literatures, in contrast, have tended to emphasize the emancipatory potential of bottom-up struggles seeking territory for autonomy. This paper advances on the understanding of territory as a set of entangled and overlapping strategies and, in doing so, moves away from attempts to conceptualize it through a bottom-up (e.g. social movements) versus top-down (e.g. the state) dichotomy. In order to do so, recent literature on socioterritorial movements -movements for whom territory is central to their strategy- and some of the overlapping and entangled power
\end{abstract}


El Territorio en disputa: estrategias políticas y...

SAM HALVORSEN

relations will be examined and explored. The article is organized in three sections, which corresponds to particularly visible strategies of socioterritorial movements in the region from recent decades.

KEYWORDS: TERRITORY. STRATEGY. SOCIOTERRITORIAL MOVEMENTS. POWER RELATIONS. LATIN AMERICA.

PALAVRAS-CHAVE: TERRITÓRIO. ESTRATÉGIA. MOVIMIENTOS SOCIOTERRITORIAIS

RELAÇÕES DE PODER. AMÉRICA LATINA.

\section{Introducción}

El territorio es una idea y práctica fuertemente disputada. En las literaturas anglosajonas los debates se han enfocado en poner en cuestión las suposiciones de que el territorio es un "contenedor" fijo y soberano (Agnew, 1994), organizado a una sola escala (Brenner, 1999), y han entendido la producción de territorios a través de redes y relaciones extra-locales (Painter, 2010). Sin embargo, como muestra la genealogía de Stuart Elden (2010:322), el territorio suele entenderse como "un espacio limitado bajo el control de un grupo de personas, normalmente un estado". En este sentido, el concepto anglosajón hace mucho hincapié en las estrategias territoriales producidas desde arriba. En contraste, diversas literaturas latinoamericanas prestan atención a las múltiples estrategias de "reinvención" del territorio colonial (Porto Gonçalves, 2012), subrayando la importancia de la autonomía y la autogestión basada en relaciones de poder menos jerárquicas. En ese contexto surgieron conceptos como "territorios disidentes" (Souza, 2015a), "territorios en resistencia" (Zibechi, 2007;2012), "territorios insurgentes" (Wahren, 2011), indicando una tendencia de romantizar el territorio a partir de concebirlo como parte de una estrategia emancipadora, impulsada desde abajo. Este artículo tiene el objetivo de integrar estas literaturas y así romper con la dicotomía que plantea pensar el territorio como estrategia política producida o desde arriba o desde abajo para avanzar hacia una lectura más abierta del concepto. Utilizamos la categoría de movimientos socioterritoriales, aquellas luchas que toman como estrategia central la apropriación del espacio, para ayudarnos a analizar disputas sobre el territorio en las cuales hay multiples formas y relaciones de poder.

El artículo desarrolla una definición abierta de territorio; se lo entiende como el resultado de la apropiación del espacio a través de estrategias políticas. En esta definición coexisten múltiples estrategias superpuestas e intrincadas, desde aquellas correspondientes al Estado colonial hasta las búsquedas de autonomía y emancipación de pueblos originarios en Abya Ayala/ América Latina. De esta manera, se intenta mostrar que el territorio está atravesado por múltiples relaciones de poder y que distintas ideas y prácticas territoriales están relacionadas internamente.

Específicamente, el articulo considera diversos ejemplos de movimientos socioterritoriales de América Latina para demostrar cómo las estrategias territoriales se intrincan y sobreponen en el proceso de apropiar al espacio. Subrayamos que esta intrincación de estrategias territoriales es dada tanto por las apropiaciones históricas del espacio como por las apropiaciones simultáneas de otros actores. O sea, nuestra definición del territorio es de un proceso abierto en sentido histórico y geográfico. Las múltiples estrategias de apropiación del espacio producen un proceso continuo de territorialización, desterritorialización y reterritorialización. 
El Territorio en disputa: estrategias políticas y...

SAM HALVORSEN

En lo que sigue, proponemos un breve estado de la cuestión de las literaturas anglosajonas sobre territorio, considerando los debates principales y subrayando sus limitaciones. Luego, empezando a integrar perspectivas latinoamericanas, desarrollamos nuestra definición del territorio, como la apropiación del espacio por estrategias políticas (múltiples estrategias intrincadas y superpuestas). El resto del artículo se divide en tres partes, cada uno considerando algunas estrategias importantes de movimientos socioterritoriales: movimientos indígenas y afrodescendientes luchando por la autonomía territorial; movimientos socioterritoriales urbanos, y luchas para descolonizar el territorio del Estado.

\section{El concepto de territorio}

Uno de los trabajos más importantes que ha abordado el concepto de territorio dentro de la geografía humana anglosajona es el de Stuart Elden (2013:322), quien lo define como "un espacio demarcado bajo el control de un grupo de personas, normalmente un estado". Otros textos claves, como los de Cox (2002), Storey (2012) y Delaney (2005:2009) abren ciertos debates, por ejemplo, establecen la tensión entre el territorio como una categoría fija o como flujo. A pesar de la lectura más abarcadora de Delaney (2009), que dialoga con el concepto de territorialidad (Soja, 1971; Sack, 1986), la lectura dominante del territorio sigue entrampada en una mirada que lo ve como el resultado de un proceso desde arriba determinado, en gran parte, por las relaciones de poder del Estado moderno y eurocéntrico.

Ya en la década de 1990, el trabajo de John Agnew (1994), alertó sobre el peligro de la "trampa territorial". De acuerdo con su perspectiva, ciertos procesos (por ejemplo, los tecnológicos), que en realidad no encajan con la escala y las fronteras delimitadas del Estado-nación, quedan "atrapados" en su territorio por un centrismo-metodológico y analítico que otorga privilegio al análisis del estado moderno (ver también Taylor, 1994). Aunque Agnew y Corbridge (1995:13-15) demuestra cierta consciencia sobre el eurocentrismo que atraviesa su definición de territorio y las "ontologías espaciales" del poder, no hay casi ningún intento de repensar la categoría de territorio más allá de sus fronteras.

Debates subsiguientes sobre cómo la globalización reformuló y reestructuró al Estado moderno (p. ej. Brenner, 1999; Swyngedouw, 2004) hicieron mucho hincapié en la re-escalarización del territorio estatal (tanto a niveles supra-nacionales como subnacionales). Mientras estas literaturas contribuyeron a reconocer la forma móvil y fluida del territorio, su base epistemológica quedó intacta y fue escaso el interés por criticar la comprensión de la construcción del territorio como una estrategia del Estado desde arriba, lo que lleva a pensarlo como "espacio abstracto" (Lefebvre, 1991) enraizado en prácticas coloniales de ocupación y control.

En los últimos veinte años los debates en el mundo anglosajón sobre el territorio siguieron avanzando, pero todavía sin romper con la mirada de su configuración por estrategias estatales desde arriba. En el contexto del giro relacional e influenciado por el trabajo de Doreen Massey (2005), Joe Painter (2010) demuestra que se puede considerar al territorio como un efecto de las redes. Es decir, en vez de concebirlo como un espacio delimitado y fijo, el territorio es sólo una apariencia de algo rígido que depende 
El Territorio en disputa: estrategias políticas y...

SAM HALVORSEN

constantemente del trabajo de distintas redes (y sus actores) que mantienen y producen el territorio del Estado. Es importante mencionar que Painter (2010) explícitamente reconoce la existencia de otras tradiciones epistemológicas sobre el territorio, y dialoga en particular con la francófona. Sin embargo, él ve pocas posibilidades de integrar otras tradiciones en su conceptualización de base anglosajona y prefiere mantener una definición más estricta, vinculada al Estado y a sus estrategias de poder.

Por su lado, en 2013 Stuart Elden elaboró una genealogía del concepto del territorio en el pensamiento político occidental. Elden (2013) argumenta que es conveniente pensar al territorio como una "tecnología política" que surgió en un momento geográfico e históricamente específico y que guarda relación con avances importantes en, por ejemplo, las técnicas de la cartografía y la legalidad. El territorio, como concepto y cómo práctica, necesitaba de ciertas condiciones ontológicas para poder existir. Una gran fortaleza de su trabajo reside en la importancia que le otorga a la especificad tanto geográfica como histórica en la producción del territorio. Sin embargo, al definir territorio de esta manera limita las posibilidades de pensarlo de manera más abierta, tomando en cuenta una mayor diversidad de estrategias, incluyendo aquellas impulsadas/promovidas desde abajo. Por ende, la propuesta de Elden choca bruscamente con las ideas y experiencias de la América Latina contemporánea.

El territorio ha sido fuertemente disputado durante la historia (pos)colonial de Abya Ayala/América Latina. En los últimos 500 años los territorios de la mayoría de los pueblos originarios fueron ocupados, fragmentados (mediante la definición de las fronteras modernas) y forzados violentamente a asimilarse con el Estado moderno. $\mathrm{Al}$ mismo tiempo, enormes contingentes de población africana fueron desraizados de sus patrias y esclavizados en los Estados coloniales. Los territorios coloniales fueron impuestos a los pueblos originarios y afrodescendientes a través de clasificaciones raciales fundadas en principios como la "pureza de sangre" (Mignolo, 2003). Sin embargo, desde el principio, las prácticas de colonización occidental se han encontrado con procesos de resistencia y reclamos territoriales, mostrándose así la otra cara de la estrategia territorial de los Estados.

Las estrategias territoriales impulsadas desde abajo abundan en la historia de la región. Los levantamientos de los Mapuche en los siglos XV y XVI, que llevaron a la corona española a reconocer su gobierno autónomo territorial (Llancaqueo, 2006), o los quilombos organizados por los esclavos que escapaban de las plantaciones o minas en Brasil (Anjos, 2009) son una muestra de ello. Desde las décadas de 1970 y 1980 la idea y práctica de territorio ha sido profunda y explícitamente reinventada por parte de los movimientos sociales en la región (Porto Gonçalves, 2012). Movilizaciones como la marcha para la dignidad y territorio en 1990 en Bolivia, la marcha para el territorio y un Estado plurinacional en Ecuador en 1992 o los territorios rebeldes ocupados por el Ejercito Zapatista Nacional de Liberación en 1994 adquirieron una significatividad histórica en las luchas por el territorio desde abajo. Aunque cada disputa tiene su propia y única genealogía, el territorio se ha convertido en un eje central -tanto en la práctica como en el discurso- de las reivindicaciones de muchos movimientos sociales Latinoamericanos en las últimas décadas (Zibechi, 2007, 2012).

Una fortaleza de las literaturas latinoamericanas sobre el concepto ha sido su abordaje como categoría de la práctica, lo cual reconoce la larga historia de territorialización 
El Territorio en disputa: estrategias políticas y...

SAM HALVORSEN

de las luchas en la región y, por ende, subraya la multiplicidad de territorios que se disputan (Haesbaert, 2020; ver también a López Sandoval et al., 2017). Como sostiene Souza (1995), el territorio se constituye por múltiples relaciones de poder, incluyendo a las del Estado moderno y su soberanía, aunque no limitadas a ellas. En este artículo estamos interesados, específicamente, en las apropiaciones conscientes que tienen uno o más objetivos políticos, y que llamamos estrategias territoriales. Para avanzar en esta línea interpretativa, la próxima sección propone una definición más abierta del territorio para luego analizar algunos ejemplos de América Latina.

\section{Territorio como estrategia política intrincada y superpuesta}

Definimos al territorio como la apropiación del espacio a través estrategias políticas, que necesariamente están intrincadas y superpuestas. La apropiación se entiende como el proceso de producir prácticas e ideas que requiere la inserción de relaciones de poder en el espacio (Lefebvre, 1991; Raffestin, 1980; Soja, 1996). Sin embargo, mientras la apropiación podría provenir desde arriba o abajo, es decir, desde individuos o grupos que tienen objetivos más o menos jerárquicos y con intenciones de dominar o emancipar, es importante entender la manera en la cual las estrategias políticas se encuentran intrincadas y superpuestas en el proceso de apropiación del espacio. Territorio es un proceso de apropiación -no es algo estático-. Es producto de diversas estrategias de poder que se encuentran intrincadas y superpuestas. El territorio, por eso, siempre es multidimensional, muchas veces contradictorio.

Por un lado, el territorio no se produce en abstracto, sino en momentos y espacios específicos. Esta producción necesariamente se superpone a las apropiaciones hegemónicas del espacio ya efectivizadas por parte del Estado nación y por las relaciones sociales capitalistas (Lefebvre, 1991; Soja, 1996). Como explica Milton Santos (2000), el territorio siempre es resultado de una (re)apropiación, reproduciendo y modificando las "configuraciones territoriales" que incluyen diversas prácticas, ideas, instituciones, etc. que se inscriben en el espacio. Por lo tanto, la producción del territorio siempre se superpone con otras apropiaciones de modo tal que hay una coexistencia de múltiples territorios (superpuestos en redes y a distintas escalas), formando parte de lo que Haesbaert (2004) llama "multi-territorialidad". En este sentido, cualquier intento de apropiación del espacio casi siempre se confronta con las ideas y prácticas dominantes del territorio estatal (Elden, 2013), cuya formación va aparejada de prácticas violentas de colonización sobre la base de categorizaciones raciales que de alguna manera las justifican (Mignolo, 1995) y de desigualdades de género y clase social (Lander, 2000; Lugones, 2010; Wainwright, 2008).

Por el otro lado, el territorio está intrincado a través de las distintas estrategias que se llevan adelante en el proceso de apropiación del espacio. Históricamente, las estrategias territoriales desde abajo han sido una respuesta a los procesos violentos de desposesión y expulsión que produjeron territorios fragmentados e híbridos como una forma de supervivencia. Ejemplo de ello serían los quilombos conformados por los esclavos fugitivos en Brasil o los territorios configurados por las comunidades indígenas desplazadas de sus tierras hacia la ciudad. Más recientemente, la idea y práctica del territorio fueron explícitamente redefinidas y "reinventadas" (Porto Gonçalves, 2012), por ejemplo, a través de las estrategias legales y cartográficas orientadas a reclamar 
El Territorio en disputa: estrategias políticas y...

SAM HALVORSEN

la propiedad y el acceso a las tierras ancestrales por parte de los pueblos originarios y movimientos afrodescendientes (Bryan, 2012). En el proceso de lucha, muchas veces las estrategias "desde abajo" se intrincan explícitamente con las prácticas y discursos dominantes en el proceso de lucha (Moore, 2005). Pero también este intrincamiento puede tener lugar a partir de la co-existencia de procesos de dominación y la resistencia (Sharp et al., 2000) dentro de cualquier espacio apropiado. Hay múltiples jerarquías internas (por ejemplo, de raza, género y clase) que se reproducen dentro de las estrategias subalternas; lo que algunos entienden como "el colonialismo interno" (Mignolo, 2003; Rivera Cusicanqui, 2010).

La intrincación de las estrategias de apropiación del espacio es tanto una decisión explícita de los actores sociales (en este articulo hacemos hincapié en los movimientos socioterritoriales) como un resultado implícito de sus acciones. Es decir, a veces los actores están conscientes de la intrincación de sus estrategias territoriales con otros actores, y todos los dilemas que eso trae, pero no siempre es el caso. Además, hay casos donde movimientos socioterritoriales intentan apropiar el espacio de manera que se mantenga una autonomía de otros actores, aunque, como sostiene el artículo, dicha autonomía existe más como un deseo que como una realidad. Así, un aporte central del artículo es la importancia de reconocer la forma multidimensional (muchas veces hibrida y paradójica) de las estrategias territoriales, que absorben distintas prácticas y relaciones de poder en el proceso de territorialización, desterritorialización y reterritorialización (TDR). Este concepto de territorio está en deuda con las literaturas latinoamericanas geográficas (Haesbaert, 2004; Fernandes, 2005; Fuini, 2017; López Sandoval et al., 2017). Sin embargo, el artículo ni rechaza ni excluye las literaturas anglosajonas, enfocadas centralmente en las estrategias territoriales de dominación por el Estado moderno, justamente dada la co-existencia de las epistemologías y prácticas eurocéntricas en el contexto latinoamericano.

En resumen, en el proceso de apropiación del espacio y a través de las estrategias de distintos proyectos políticos se produce un territorio superpuesto e intrincado. Ello nos lleva a no mantener una división conceptual estricta entre el territorio y la territorialidad. En las literaturas anglosajones hay una tradición de trabajar el concepto de territorialidad como una característica humana universal (Soja, 1971), definida en un texto clásico de Sack (1986:5) como "una estrategia geográfica poderosa" que intenta controlar un área delimitada. Según Elden (2013), el territorio es un concepto más específico, en el sentido de que solo pudo existir a partir de ciertas tecnologías políticas modernas, incluyendo la cartografía y la legalidad. Sin embargo, el concepto dominante anglosajón del territorio como estrategia da el protagonismo, únicamente, al Estado moderno europeo (Brenner y Elden, 2009; Elden, 2013). Para poder ampliar el concepto como estrategia proponemos acá incorporar la idea de movimiento socioterritorial. De esta manera nos distanciamos de la literatura anglosajona y tomamos una categoría producida en América Latina que tiene la capacidad de referirse a las múltiples estrategias políticas por las cuales la apropiación del espacio es una característica fundamental.

El concepto de movimiento socioterritorial fue elaborado por Bernardo Mançano Fernandes $(2000,2005)$ no solamente para indicar la inseparabilidad de lo espacial y lo social, sino también para referirse al proceso de TDR en el cual los actores luchan, a través de formas tanto materiales como inmateriales, para alcanzar sus diversos objetivos. Como afirma Fernandes (2005:24): 
El Territorio en disputa: estrategias políticas y...

SAM HALVORSEN

Para alcanzar sus objetivos los movimientos socioterritorales construyen espacios políticos, se espacializan y promueven espacialidades. La construcción de un tipo de territorio significa, casi siempre, la destrucción de otro tipo de territorio, de modo que la mayor parte de los movimientos socioterritoriales se forman a partir de procesos de territorialización y desterritorialización (la traducción del autor).

Lo importante de esta conceptualización es que un movimiento socioterritorial implica un proceso continuo (de TDR) en el cual el territorio, como espacio apropiado, es siempre modificado y reapropiado. Fernandes (2005) sostiene que la producción de un territorio implica la "destrucción" de otro, lo cual hace más complicada la co-existencia de múltiples territorios superpuestos e intrincados (cf Haesbaert, 2004). Sin embargo, el territorio rara vez es en sí mismo "destruido", sino que en el proceso de TDR es reconfigurado, quizás cambiando la escala o el enfoque estratégico. Halvorsen et al. (2019) proponen que los movimientos socioterritoriales pueden ser analizados a partir de cuatro ejes: estrategia, identidad, socialización política e institucionalización. Estos ejes muestran que los movimientos socioterritoriales no se movilizan desde abajo o desde arriba, sino en un contexto relacional y por distintas escalas e instituciones. Esto se observa en las últimas tres décadas en algunos casos emblemáticos de movimientos socioterritoriales en América Latina.

\section{Coexistiendo con el Estado: movimientos indígenas y afrodescendientes}

Una estrategia central de los movimientos indígenas y afrodescendientes ha sido la búsqueda de alguna forma de autonomía territorial (Bryan, 2012; Escárzaga et al., 2014; González, 2015; Sesia y Sarmiento, 2008). Por un lado, los movimientos socioterritoriales han dependido de las prácticas e ideas modernas del Estado nación para poder garantizar su autonomía de manera legal e institucional (Zuñiga Navaro, 1998). Muchas veces, la autonomía territorial ha sido construida adentro de los reclamos soberanos del Estado sobre el espacio, mediante el reconocimiento jurídico de los derechos a la auto-determinación de los pueblos indígenas y afrodescendientes, en particular por vía de reformas en la Constitución y de las titulaciones de las tierras (Bryan, 2012; González, 2015; Offen, 2003), lo que ha producido "territorialidades superpuestas" (Agnew y Oslender, 2013). Esto abrió nuevas oportunidades para los reclamos noestatales, pero también encontró límites. A pesar de valerse de saberes alternativos (por ejemplo, la gobernanza colectiva y los derechos comunales de tierra), el recurso a prácticas territoriales legales (derechos constitucionales) y cartográficos (mapeo de los títulos de tierra) llevó a un enredo con ideas y estrategias coloniales en relación al territorio (Wainwright y Bryan, 2009). Es decir, para lograr cierta forma de autonomía los movimientos socioterritoriales suelen usar las "tecnologías políticas" (Elden, 2013) como la cartografía, y así caen en un proceso de territorialización que proviene de las epistemologías coloniales. Por lo tanto, distintas estrategias territoriales se intrincan en el proceso de buscar autonomía.

Al mismo tiempo, los reclamos de autonomía territorial se han servido de las oportunidades creadas por la reestructuración neoliberal de la década de 1990. Por ejemplo, en gran parte de América Central, la presión del Banco Mundial llevó a reformas de 
El Territorio en disputa: estrategias políticas y...

SAM HALVORSEN

tierra (estas fueron vendidas a los sectores privados, algunos de ellos transnacionales). Este proceso fue paralelo al "giro" multicultural que supuso la ampliación de derechos de pueblos indígenas y de afrodescendientes (Larson et al., 2016). Ambas dinámicas se desarrollan bajo una lógica territorial moderna de parcialización y privatización. Esto produjo que las estrategias de autonomía territorial en muchos Estados centroamericanos (y en otros lados) se intrincaran fuertemente con las "mismas estructuras de dominación que estas comunidades intentaron resistir" (Hale, 2011:7). Este "giro" territorial" en los reclamos de autonomía indígenas y afrodescendientes, basado en la auto-gobernanza y en los derechos colectivos, ha quedado atrapado entre las tecnologías políticas hegemónicas del Estado y las reformas neoliberales basadas en la propiedad privada (Bryan, 2012). Además, como subraya Ulloa (2011, 2012), los movimientos indígenas colombianos al construir la autonomía territorial suelen requerir la negociación con otros territorios superpuestos, incluyendo los de los narcotraficantes, los paramilitares, las organizaciones guerrilleras y las ONG internacionales. Ello requiere de cierta internalización de distintas formas de entender el territorio que entran en disputa (Ballvé, 2012; Courtheyn, 2018). Es decir, los movimientos socioterritoriales pueden tomar una decisión estratégica de internalizar lógicas territoriales de dominación para poder dialogar y negociar con otros actores y, en particular, con las instituciones estatales.

Por otro lado, la autonomía territorial ha sido construida en resistencia directa a las estrategias dominantes del territorio. El ejemplo más prominente es el de los zapatistas que, luego del fracaso en el intento de llegar a un acuerdo con el gobierno (en los acuerdos de San Andrés, 1996), rechazaron por completo la soberanía del Estado mexicano (Reyes y Kaufman, 2011). La autonomía territorial de los zapatistas se puede entender como una negación de las estrategias de "toma del poder" (Holloway, 2002) y un intento de construir relaciones de poder horizontales y no-jerárquicas (Baronnet et al., 2011; Dinerstein et al., 2013; Holloway y Peláez, 1998), enraizadas en las prácticas cotidianas como la producción de alimentos (Naylor, 2017). A pesar de la resistencia de muchos zapatistas a cualquier tipo de soberanía, el Estado está presente dentro de los territorios rebeldes, más claramente bajo la forma de los controles militares, pero también en prácticas cotidianas como el financiamiento de las escuelas o la representación legal. Esta presencia provoca ciertas tensiones dentro de las comunidades diversas de Chiapas (Barmeyer, 2008; Mora, 2015). Más recientemente, es destacable que los zapatistas presentaron una candidata, María de Jesús Patricia, para las elecciones presidenciales en 2018. Los territorios autónomos de los movimientos socioterritoriales se presentan como contrapunto al poder soberano estatal, pero deben entenderse en el contexto de la hegemonía persistente de las ideas coloniales de territorio. En este marco, las estrategias de los movimientos socioterritoriales por la autonomía mantienen relaciones internas con las estrategias de dominación estatal, lo cual tiende a producir dilemas.

\section{Movimientos socioterritoriales urbanos: estrategias de sobrevivencia}

La ciudad latinoamericana es, cada vez más, un terreno de lucha central para diversos movimientos socioterritoriales que buscan la autonomía para poder apropiarse del espacio en el contexto de la presencia informal del Estado combinado con las prácticas neo-coloniales de la "acumulación por desposesión" urbana (Harvey, 2012; Lees et 
El Territorio en disputa: estrategias políticas y...

SAM HALVORSEN

al., 2015; Souza, 2015a). Es decir, la centralidad del territorio urbano para la acumulación capitalista en las últimas décadas ha provocado fuertes resistencias de nuevos movimientos socioterritoriales intentando sobrevivir en los márgenes y reclamar sus “derechos a la ciudad" (Lefebvre, 1969).

El Gran Buenos Aires ejemplifica la "inscripción territorial" (Merklen, 2005) de las clases populares que se apropiaron del espacio urbano como una forma de sobrevivencia y organización política. Tras la desindustrialización pos 70 y los años de la dictadura (1976-1983), disminuyeron las fábricas y sindicatos (además de los partidos políticos) como espacios de la organización política, y el neoliberalismo tomó centralidad en las ideas y prácticas del desarrollo del país. En este contexto los barrios obreros, que en algunos casos empezaron como asentamientos, se volvieron sitios claves para la construcción de nuevas identidades y organizaciones políticas (Grimson et al., 2009; Svampa y Pereyra, 2003; Tobío, 2012). Durante los años 90 los trabajadores desempleados reposicionaron la táctica del piquete más allá de la fábrica y hacia el corte de las rutas como forma de huelga, apropiándose simultáneamente del espacio urbano por su "trabajo territorial" (Delamata, 2004, 2005; Manzano, 2015; Mazzeo, 2014; Menazzi, 2008; Torres, 2011). Este giro territorial en las estrategias de los movimientos populares urbanos dio visibilidad a sus luchas, pero también produjo nuevas formas de pensar y producir el territorio.

El término trabajo territorial fue propuesto como una manera de redefinir el valor de la mano de obra pos-industrial, comprender su pasaje desde la fábrica y otorgarle su valor social en el barrio -la "nueva fábrica" (Stratta y Barrera, 2009)- como una forma de vivir con dignidad (Dinerstein, 2014). Además, el trabajo territorial permitió la organización de la producción de poder popular y el desarrollo de relaciones de poder “autónomas y horizontales" (MTD Almirante Brown, 2002; Mazzeo et al., 2007) a través de prácticas cotidianas como los comedores, los centros de salud, las actividades artísticas y las economías solidarias (Farías, 2018). Estos ejemplos demuestran los intentos por redefinir el territorio a partir de formas de vivir no-jerárquicas, anti-estatistas y anti-capitalistas (Dinerstein, 2015). Sin embargo, también demuestran que mucho de estos territorios autónomos urbanos se configuraron en interacción con las estrategias estatales de controlar el espacio a través de las prácticas clientelistas de gobiernos populistas. Por ejemplo, el gobierno progresista kirchnerista (2003-15) intentó incorporar ciertos movimientos urbanos socioterritoriales bajo sus estructuras de gobernanza local (Natalucci et al., 2013; Rossi, 2015, 2017) disminuyendo las distinciones entre las estrategias territoriales desde arriba y desde abajo y abriendo debates sobre los peligros de institucionalizar la acción colectiva (Frederic, 2004; Manzano, 2007; Quirós, 2008; Forni y Castronuovo, 2013; Massetti et al., 2010; Nataulcci y Pérez, 2015; Retamozo et al., 2013). Las relaciones entre movimientos socioterritoriales y gobiernos han sido paradójicas en los últimos años, mostrando tanto colaboración como represión. En ciertos casos las distinciones entre movimiento y gobierno (o partidos políticos) se han colapsado debido a su fuerte intrincamiento (Bringel y Falero, 2016), mientras en otros casos se ha mantenido una división fuerte entre estos actores (Zibechi, 2007, 2012).

La ocupación de tierras en las ciudades latinoamericanas también demuestra la coexistencia de diversas estrategias territoriales, particularmente visibles en las villas/ favelas urbanas. Las favelas/villas latinoamericanas representan territorios paradójicos e híbridos que cambian en el espacio y en el tiempo (p. ej. Clichevsky, 2003). 
El Territorio en disputa: estrategias políticas y...

SAM HALVORSEN

Desde la perspectiva de la planificación urbana, por ejemplo, ciudades como Buenos Aires demuestran ciclos alternantes de integración y expulsión con/desde el espacio urbano "formal" (Cravino, 2009; Di Virgilio et al., 2014; Massidda, 2018). Desde una perspectiva desde abajo, la década de 1990 vivenció un cambio en muchas ciudades latinoamericanas hacia la movilización por títulos de propiedad de parte de los habitantes de villas que, en paralelo a las experiencias de comunidades indígenas, han tendido a debilitar la solidaridad colectiva y las relaciones con la tierra (Borrell, 2016; Varley, 2017). La villa, por tanto, representa un territorio híbrido y paradójico (Varley, 2013) que plantea desafíos para cualquier análisis, tal como nos muestran las experiencias bien documentadas de la favela brasileña.

Por un lado, la favela reproduce y sostiene las relaciones territoriales coloniales basadas en la desigualdad socioeconómica (tal como suministrar mano de obra barata) y la exclusión política (por ejemplo, ausencia del Estado de bienestar o la representación institucional) (Barbosa y Silva, 2013; Muxica, 2011; Leite, 2008). Perlman (1976) denunció el famoso mito de la marginalidad en las favelas de Río de Janeiro y sostuvo que el problema no era una falta de integración a la ciudad, sino la forma explotadora de relacionarse con los proyectos políticos dominantes (por caso, la mano de obra subpagada, las relaciones clientelistas, las culturas estigmatizadas). Al mismo tiempo, el Estado impuso, violentamente, la lógica de su forma de apropiación del espacio dominante sobre la favela, por ejemplo, en las prácticas de construcción de fronteras/ murallas que material y simbólicamente controlan las agencias de los habitantes de favelas (Burgos, 2005; Haesbaert, 2014) o la militarización de favelas en el contexto de "pacificar" las organizaciones narco-traficantes (Leite, 2012), formando así "territorios de miedo" (Souza, 2008).

Por otro lado, las favelas demuestran la potencia de los movimientos urbanos socioterritoriales que, a través de sus estrategias, intentan romper con las lógicas de territorialización dominantes. Las experiencias compartidas y encarnadas de convivencia en la proximidad han producido numerosas oportunidades para los proyectos colectivos y de base (Barbosa y Silva, 2013). Por ejemplo, los pueblos afrodescendientes han (re) producido la favela como un sitio simbólico de la identidad subalterna, a través de prácticas culturales como el rap, subrayando las (in)justicias territoriales/raciales y afirmando las subjetividades políticas negras y las relaciones sociales no opresivas (Da Silva, 2009; Pérez-Wilke, 2014). Estas subjetividades radicales, a menudo entendidas explícitamente en referencia a los quilombos históricos y a las tierras ancestrales en África (Carril, 2006; Pérez-Wilke y Marquez, 2013), simbólicamente resisten e interrumpen en el "blanqueamiento" neo-colonial del espacio urbano en Brasil (Correa, 2017). Además, la exclusión política formal de los favelados ha producido lo que Holston (2008) llama una "ciudadana insurgente" basada en formas innovadoras de construir las relaciones institucionales con el Estado a partir de la organización de base. La favela así demuestra potencia para generar lo que Souza (2015a) llama "territorios disidentes": experimentos insurgentes con relaciones sociales, económicas y políticas alternativas.

Aunque no está ubicado formalmente en la favela, el movimiento sem teto (sin techo) demuestra la potencia de valerse de las ocupaciones urbanas, particularmente de los edificios, para construir enlaces entre las luchas de los favelados y otros movimientos sociales, mientras también experimentan con prácticas emancipadoras como el trabajo cooperativo (Souza, 2015a, 2015b). No obstante, una demanda clave de los favelados 
El Territorio en disputa: estrategias políticas y...

SAM HALVORSEN

en este y otros movimientos es la mayor inclusión en los territorios urbanos formales (Falcão y Falbo, 2016; Silva, 2008; Souza, 2015a, 2015b). De esto surgen nuevas preguntas sobre el rol clave del Estado en las estrategias territoriales, algo que ha sido central a los debates contemporáneos en toda América Latina.

\section{Descolonizando el territorio del Estado en Bolivia y Ecuador}

A partir de los principios de los años 2000 ha habido intentos explícitos de disputar y descolonizar el territorio construido por el Estado nación a través de las victorias electorales y las reformas constitucionales realizadas en algunos países de América Latina, sobre todo en Bolivia y Ecuador. La victoria de Evo Morales como el primer presidente indígena de Bolivia en 2005 abrió un proceso participativo (la asamblea constituyente) orientado a reescribir la constitución en la cual la idea de un "Estado plurinacional" se hizo una columna central (Lizárraga, 2009; Rivero, 2014). Bolivia, donde la mayoría de la población es indígena, estuvo durante mucho tiempo gobernada por una minoría racial cuyo Estado dio poco reconocimiento a los múltiples territorios de sus habitantes originales, produciendo un territorio "abigarrado" (Tapia, 2002) con un Estado monocultural supra-impuesto (desde arriba): una relación de dominación y desarticulación (Rivero, 2014). La nueva constitución intentó romper con la hegemonía del territorio colonial, identificando los derechos de los pueblos indígenas a la gobernanza autónoma del territorio, incluyendo el uso de recursos (Lizárraga y Rivero, 2014; Rivero, 2014). Un proceso parecido se desarrolló en Ecuador durante la gestión del Presidente Rafael Correa (2007-2017). Igual que en Bolivia, la nueva constitución (2008) no solamente hizo referencia al Estado plurinacional, sino también al concepto de buen vivir/sumac kawsay (coexistencia, diversidad y armonía con la naturaleza) (Radcliffe, 2012; Restrepo y Valencia, 2016; Varea y Zaragocin, 2017; Walsh, 2010). Aunque intentos previos de reformas constitucionales habían hecho algún progreso hacia la implementación de la autonomía territorial indígena (ver González, 2015), Ecuador y Bolivia representan intentos radicales de incorporar comprensiones indígenas del territorio en la estructura legal-institucional del Estado.

A pesar de estos avances, los Estados plurinacionales de Bolivia y Ecuador siguen atrapados de distinta manera dentro de un sistema territorial dominante. Por ejemplo, ambos continúan existiendo dentro de las fronteras modernas impuestas y están gobernados por sistemas electorales modernos dominados por los partidos políticos (Lizárraga, 2009). Luego de la victoria de Correa en las elecciones, algunos movimientos socioterritoriales, que apoyaron su llegada al poder, al poco tiempo mostraron discrepancias con el líder político y decidieron alejarse del gobierno. A su vez, los líderes indígenas tuvieron poco peso en la gobernanza nacional o local (Radcliffe, 2012). Una crítica importante realizada a estos gobiernos andinos pos-neoliberales es el hecho que en el marco del Consenso de los Commodities (Svampa, 2015) las exportaciones de materias primas se usaron para subsidiar las políticas de redistribución social. Esto produjo un giro "ecoterritorial" en algunos los movimientos socioterritoriales en respuesta a la "explosión de las luchas socioambientales" generadas en las comunidades rurales e indígenas (Svampa, 2017:80). Un ejemplo clave en Bolivia fue el conflicto TIPNIS en el cual la propuesta del gobierno para construir una ruta que atravesaba tierras indígenas enfrentó una oposición y conflicto fuerte, exponiendo límites reales a la descolonización de la gobernanza estatal-territorial (Gudynas, 2013; Hirsch, 2019; 
El Territorio en disputa: estrategias políticas y...

SAM HALVORSEN

Hope, 2014; Rosell Arce, 2012). Las resistencias antiextractivistas, que se inspiraron en una comprensión del territorio basadas en la gobernanza colectiva y las interpretaciones de los pueblos originarios sobres las relaciones sociedad-naturaleza, se consideraron en contra de los intereses nacionales y fueron clasificados por el vicepresidente Álvaro García Linera como "colonialismo ambientalista" (Svampa, 2017).

Las luchas por la tierra desafían las epistemologías modernas de territorio basadas en la propiedad privada y en la concentración de los derechos. También desafían la racionalidad cartesiana que separa los procesos sociales y naturales y reduce a la tierra a sus dimensiones objetivas y cuantificables. Muchas comunidades indígenas comprenden y producen territorio a través de diversas relaciones "socio-naturales" que juntan múltiples "seres animados" (como una montaña) con su propia agencia política (De la Cadena, 2015). Aquí lo que está en juego es el hacer visible y permitir la co-existencia de múltiples mundos, una "pluriversa" (De la Cadena, 2015), lo que algunos autores entienden como la "ontología política" de territorio (Blaser, 2014; Escobar, 2016). El territorio de comunidades negras en la costa Pacífica de Colombia, por ejemplo, se forma por múltiples vidas humanas y no-humanas (p. ej. manglares) dentro de un "espacio acuático" (Oslender, 2016) cuyos ritmos están profundamente entrecruzados por las interacciones sociales y las construcciones de identidades (Satizábal y Batterbury, 2018). La producción de territorio en tales contextos no solamente desafía a la fijeza de los supuestos eurocéntricos al enfatizar la movilidad/fluidez (ver también Steinberg, 2009), sino que también reconoce distintas maneras de entender y practicar el territorio, las cuales indican los límites intrínsecos a cualquier comprensión particular (Escobar, 2008, 2016; Porto Gonçalves, 2002; Ulloa, 2011). El cambio de discurso de la tierra hacia el territorio en los movimientos socioterritoriales indígenas y otros (ver Porto Gonçalves, 2012) sugiere una estrategia territorial de movilizar la multidimensionalidad en contra de sus comprensiones monolíticas y modernas (Giarracca y Teubal, 2013; Rosset, 2013).

\section{Conclusión}

Los movimientos socioterritoriales acuden a estrategias de apropiación espacial para alcanzar diversos objetivos. Es clave reconocer los límites de las formas de entender el territorio desde las perspectivas anglosajonas, que siguen entrampadas dentro de las experiencias occidentales y coloniales, pero también es necesario evitar el romanticismo que persiste en algunas comprensiones latinoamericanas (Souza, 2015a; Wahren, 2011; Zibechi, 2012). De esta manera, el concepto de movimiento socioterritorial tampoco debe ser romantizado. Sostuvimos la potencia de llevar adelante el análisis de diversas formas de movimiento, no solamente de aquellos que tienen características de ser desde abajo, y que se orientan a la autonomía territorial, sino también los que están intrincados con el Estado y provienen desde arriba. Este texto mostró, además, la necesidad de romper con ciertas dicotomías en el análisis de la política territorial, como las de política formal/no formal, de partido/movimiento y del territorio como categoría de dominación o emancipación.

El artículo tomó como base conceptual la literatura geográfica latinoamericana de los últimos años en la cual el territorio se entiende como un proceso de apropiación del espacio por relaciones de poder. Su contribución más específica fue la de definir el 
territorio como el resultado de estrategias de apropiación espacial que necesariamente terminan intrincadas entre sí. Mientras las intrincaciones de estrategias territoriales pueden ser dadas en las apropiaciones históricas del espacio, hicimos hincapié en la coexistencia simultánea de distintas ideas y prácticas del territorio. La intrincación de estrategias territoriales puede ser una decisión consciente de los movimientos socioterritoriales, incluso una necesidad de sobrevivencia, o puede ser un producto implícito del proceso de TDR. De todas maneras, el territorio se suele producir en las estrategias de movimientos socioterritoriales, de forma híbrida y paradojal, con múltiples tensiones y dilemas.

El análisis del territorio y de los movimientos socioterritoriales presentado también tiene relevancia más allá de América Latina, donde las estrategias de territorialización, desterritorialización y reterritorialización adquieren una fuerte relevancia (p. ej. Nel·lo, 2003; del Romero, 2014; Routledge, 2015). De esta forma, América Latina puede ser concebida como un importante espacio de la producción epistemológica para establecer diálogos con otras ideas y experiencias correspondientes a otros contextos epistemológicos. Por ende, recomendamos ir más allá de los análisis de movimientos socioterritoriales como un fenómeno específicamente latinoamericano y entender la importancia más universal de las estrategias territoriales. De la misma manera, es necesario entender la centralidad de las estrategias territoriales en toda organización política que necesariamente tiene que interactuar con el Estado, pero que también precisa buscar recursos e ideas a distintas escalas y a través de diversas redes. El territorio solo existe en su disputa y las estrategias de los movimientos socioterritoriales de América Latina son un buen punto de partida para empezar un dialogo más amplio.

Al autor le gustaría agradecer a Mariana Arzeno y Mónica Farías por su ayuda con la ortografía, a tres evaluadores por sus comentarios detallados los cuales mejoraron la última versión y a Ernesto Dufour por su asistencia editorial. El autor reconoce el financiamiento del Leverhulme Trust (ECF-2016-301). Cualquier responsabilidad es únicamente del autor. 


\section{Q Bibliografía}

»Agnew, J. (1994). The territorial trap: the geographical assumptions of international relations theory. Review of International Political Economy, 1(1), 5380.

» Agnew, J. y Corbridge, S. (1995). Mastering Space: Hegemony, Territory and International Relations. Lanham MD: Rowman \& Littlefield.

» Agnew, J. y Oslender, U. (2013). Territorialities, Sovereignty in Dispute: Empirical Lessons from Latin America. En W. Nicholls. B. Miller y J. Beaumont (Eds.), Spaces of Contention: Spatialities and Social Movements (pp. 121-140). Farnham: Ashgate.

»Anjos, RSA. (2009). Quilombos: Geografia Africana, cartografia étnica, territórios tradicionais. Brasília: Mapas editora e consultoria.

» Ballvé, T. (2012). Everyday state formation: territory, decentralization, and the narco landgrab in Colombia. Environment and Planning D: Society and Space, 30, 603-622.

» Barmeyer, N, (2008),.Taking on the state: Resistance, education, and other challenges facing the zapatista autonomy project. Identities: Global Studies in Culture and Power, 15(5), 506-527.

» Barbosa, J.L. y Silva, J.S. (2013). As favelas como territórios de reinvenção da cidade. Cadernos do Desenvolvimento Fluminense, 1, 115-126.

» Baronnet, B., Mora, M., y Stahler-Sholk, R. (2011). Luchas "muy otras": zapatismo y autonomía en las comunidades indígenas de Chiapas. México, D.F.; Chipas: CIESAS, Universidad Autónoma de Chiapas.

» Blaser, M. (2014). Ontology and indigeneity: on the political ontology of heterogeneous assemblages. Cultural geographies, 21(1), 49-58.

» Borrell, M.A. (2016). La ciudad dividida: el territorio de la informalidad como campo afectivo. Abriu, 5, 137-150.

» Brenner, N. (1999). Beyond state-centrism? Space, territoriality, and geographical scale in globalization studies. Theory and Society, 28, 39-78.

» Brenner, N. y Elden, S. (2009). Henri Lefebvre on State, Space, Territory. International Political Sociology, 3, 353-377.

» Bringel, B. y Falero, A. (2016). Movimientos sociales, gobiernos progresistas y Estado en América Latina: transiciones, conflictos y mediaciones. Cad. Crh [online], 29(3), 27-45. Recuperado de: http://www.scielo.br/pdf/ccrh/ v29nspe3/0103-4979-ccrh-29-spe3-0027.pdf

» Bryan, J. (2012). Rethinking Territory: Social Justice and Neoliberalism in Latin America's Territorial Turn. Geography Compass, 6(4), 215-226.

"Burgos, M.B. (2005). Cidade, territórios e cidadania. DADOS, Revista de Ciencias Sociais, 48(1), 189-222

» Carril, L.F.B. (2006). Quilombo, favela e periferia: a longa busca da cidadania. São Paulo: FAPESP 
»Clichevsky, N. (2003). Territorios en pugna: las villas de Buenos Aires. Ciudad y Territorio Estudios Territoriales, 35(136-137), 347-374

»Correa, G.A. (2017). O branqueamento do território como dispositivo de colonialidade do poder: notas sobre o contexto brasileiro.. En V.D.C. Cruz y D.A.D. Oliveira (Eds.), Geografia e Giro Descolonial: Experiencias, Ideias e Horizontes de Renovação do Pensamento Crítico (pp. 117-130). Rio de Janiero: Letra Capital.

» Courtheyn, C. (2018). Territories of peace: alter-territorialities in Colombia's San José de Apartadó Peace Community. The Journal of Peasant Studies, 45(7), 14321459.

» Cox, K.R. (2002). Political Geography: Territory, State, and Society. Oxford: Blackwell.

»Cravino, M.V. (2009). Territorialidades en las villas de la Ciudad de Buenos Aires. Estado, mercado y relaciones sociales en la especialidad barrial. En A. Catenazzi, A. Quintar, M.C. Cravino, N. Da Representação y A. Nocick (Eds.), El retorno de lo politico a la cuestión urbana: Territorialidad y acción pública en el Área Metropolitana de Buenos Aires (pp. 45-78). Buenos Aires: Prometeo Libros.

» Da Silva, A.C.C. (2009). Segregação espacial e produção de territórios negros por blocos afro em Ilheus, Bahia. Pontourbe, 4, 1-19.

»De la Cadena, M. (2015). Earth Beings. Durham: Duke University Press.

»Del Romero, L. (2014). Movimientos sociales y conflictos territoriales: el caso de Barcelona y Valencia. Cuadernos de Geografía, 94, 17-35.

»Delamata, G. (2004). Los Barrios Desbordados: Las organizaciones de desocupados del Gran Buenos Aires. Buenos Aires: Libros de Rojas.

»Delamata, G. (2005). Ciudadanía y Territorio: Las relaciones políticas de las nuevas identidades sociales. Buenos Aires: Espacio.

»Delaney, D. (2005). Territory: a short introduction. Oxford: Blackwell.

» Delaney, D. (2009). Territory and Territoriality. En R. Kitchin y N. Thrift (Eds.), International Encyclopedia of Human Geography (pp. 196-208), vol. 11. Oxford: Elsevier.

» Dinerstein, A.C., Contratese, D., Deledicque, M., Ferrero, J.P., Ghiotto, L. y Pascual, R. (2013). Movimientos Sociales y Autonomía Colectiva. Buenos Aires: Capital Intelectual.

» Dinerstein, A.C. (2014). The dream of dignified work: on good and bad utopías. Development and Change 45(5), 1037-1058.

» Dinerstein, A.C. (2015). The Politics of Autonomy in Latin America. Basingstoke: Palgrave Macmillan.

» Di Virgilio, M.M., Guevara, T.A. y Arqueros Mejica, M.S. (2014). Políticas de regularización en barrios populares de origen informal en Argentina, Brasil y México. Urbano, 17(29), 57-65.

»Elden, S. (2010). Land, terrain, territory. Progress in Human Geography, 34(6), 799817.

» Elden, S. (2013). The Birth of Territory. London: University of Chicago Press.

»Escárzaga, F., Gutiérrez, R., Carrillo, J.J., Capece, E. y Nehe, B. (2014). Movimiento indígena en América Latina: resistencia y transformación social, vol. III. Mexico: 
Centro de Investigaciones y Estudios Superiores en Antropología Social.

»Escobar, A. (2008). Territories of Difference: place, movements, life, redes. London: Duke University Press.

»Escobar, A. (2016). Thinking-feeling with the Earth: Territorial Struggles and the Ontological Dimensions of the Epistemologies of the South. Revista de Antropología Iberoamericana, 11(1), 11-32.

»Falcão, M. y Falbo, R. (2016). Quilombo das guerreiras e Zumbi dos Palmares: Movimentos sociais pelo direito a moradia na cidade do Rio de Janeiro. Direito da Cidade, 8(1), 331-360.

»Farías, M. (2018). Contesting Exclusion: Solidarity Spaces and Changing Political Subjectivities in Buenos Aires. Geoforum, Recuperado de: https://www. sciencedirect.com/science/article/abs/pii/S0016718518301404

»Fernandes, B.M. (2000). Movimento Social como Categoria Geográfica. Revista TerraLivre, 15, 59-85.

»Fernandes, B.M. (2005). Movimentos socioterritoriais e movimentos socioespaciais: contribução teórica para uma leitura geográfica dos movimentos sociais .Revista Nera, 8(6), 14-34-.

» Forni, P. y Castronuovo, L. (Eds.). (2013). Ni Piqueteros Ni Punteros: Organizaciones Populares durante el Kirchnerismo. La Plata: EDULP.

»Frederic, S. (2004). Buenos vecinos, malos políticos. Moralidad y política en el Gran Buenos Aires. Buenos Aires: Promoteo.

» Fuini, L.L. (2017). Construções teóricas sobre o território e sua transição: a contribuição da Geografia brasileira. Cuadernos de Geografía: Revista Colombiana de Geografía, 26(1), 221-242.

» Giarracca, N. y Teubal, M. (Eds.). (2013). Actividades extractivas en expansión: ¿Reprimarización de la economia argentina? Buenos Aires: Editorial Antropofagia.

» González, M. (2015). Indigenous territorial autonomy in Latin America: an overview. Latin American and Caribbean Ethnic Studies, 10(1), 10-36.

» Grimson, A. Ferraudi, C.M.C. y Segura, R. (2009). La vida política en los barrios populares de Buenos Aires. Buenos Aires: Prometeo.

» Gudynas, E. (2013). El malestar Moderno con el Buen Vivir: Reacciones y resistencias frente a una alternativa al desarrollo. Ecuador Debate, 88,183-205.

» Haesbaert, R. (2004). O mito da desterritorializção: do "fim dos territórios" a multiterritorialidade. Rio de Janeiro: Bertrand Brasil.

» Haesbaert, R. (2014). Contenção Territorial: "Campos" e Novos Muros. Boletín de Estudios Geográficos, 102, 25-45.

» Haesbaert, R. (2016). As Armadilhas do Território. En J.B. da Sliva, C.N.M. da Silva y E.W.C. Dantas (Eds), Território: Modo de Pensar e Usar (pp. 19-42). Fortaleza: Edições UFC.

» Haesbaert, R. (2020). Território(s) numa perspectiva latino-americana. Journal of Latin American Geography, 19(1), 141-151.

» Hale, C. (2011). Resistencia para que? Territory, autonomy and neoliberal entanglements in the 'empty spaces' of Central America. Economy and Society, 40(2), 184-210. 
SAM HALVORSEN

» Halvorsen, S., Fernandes, B.M. y Torres, D. (2019). Mobilising Territory: Socioterritorial movements in comparative perspective. Annals of the Association of American Geographers, 109(5), 1454-1470.

» Harvey, D. (2012). Ciudades rebeldes. Del derecho a la ciudad a la revolución urbana. Madrid, Ediciones Akal.

» Hirsch, C. (2019). Between resistance and negotiation: indigenous organisations and the Bolivian State in the case of TIPNIS'. The Journal of Peasant Studies, 46(4), 811-830.

» Holloway, J. (2002). Change the World Without Taking Power. London: Pluto Press.

» Holloway, J. y Peláez, E. (Eds.). (1998). Zapatista! Reinventing Revolution in Mexico. London: Pluto Press.

» Holston, J. (2008). Insurgent Citizenship: Disjunctions of Democracy and Modernity in Brazil. Princeton: Princeton University Press.

»Hope, J. (2014). Losing ground? Extractive-led development versus environmentalism in the Isiboro Secure Indigenous Territory and National Park (TIPNIS), Bolivia. The Extractive Industries and Society, 3(4), 922-929.

»Lander, E. (Ed.). (2000). La Colonialidad del Saber: Eurocentrismo y Ciencias Sociales. Buenos Aires: CLASCO.

»Larson, A.M., Soto, F., Mairena, D., Moreon, E., Mairena, E. y Mendoza-Lewis, J. (2016). The Challenge of 'Territory': Weaving the Social Fabric of Indigenous Communities in Nicaragua's Northern Caribbean Autonomous Region. Bulletin of Latin American Research, 35, 322-337.

» Lees, L., Shin, H.B. y López-Morales, E. (2015). Global gentrifications: Uneven development and displacement. Bristol: Policy Press.

» Lefebvre, H. (1969). El derecho a la Ciudad. Barcelona: Ediciones Península.

» Lefebvre, H. (1991) 2013. La producción del espacio. Madrid: Capitán Swing.

》 Leite, M.P. (2008). Pobreza y exclusión en las favelas de Río de Janeiro. En L. Ziccardi (Ed.), Procesos de urbanización de la pobreza y nuevas formas de exclusión social: los retos de las políticas sociales de las ciudades latinoamericanas del siglo XXI (pp. 213-247). Bogotá: CLACSO

» Leite, M.P. (2012). Da "metáfora da guerra" ao projeto de "pacificação": favelas e políticas de segurança pública no Rio de Janeiro. Revista Brasileira de Segurança Publica, 6(2), 374-389.

》 Lizárraga, P. (2009). La descolonización del territorio: Visiones de país y la configuración del Estado Plurinacional en Bolivia. IV Jornada de Estudos em Assentamentos Rurais. Recuperado de: http://www.uff.br/vsinga/trabalhos/ Trabalhos\%20Completos/Claudia\%20Pilar\%20Liz\%E1rraga\%20Aranibar.pdf

» Lizárraga, P. y Rivero, C.V. (2014). La descolonización del territorio: luchas y resistencias campesinas e indígenas en Bolivia. En G. Almeyra, L.C. Bórquez, J.M. Pereira y C.W. Porto-Gonçalves (Eds.), Capitalismo: Tierra y poder en América Latina (pp. 1982-2012), vol. II. Mexico DF: CLACSO.

»Llancaqueo, V.T. (2006). Pueblo Mapuche. Derechos Colectivos y Territorio: Desafíos para la sustentabilidad democrática. Programa Chile Sustentable: e-book.

» López Sandoval, M.F., Robertsdotter, A. y Paredes, M. (2017). Space, Power and 
Locality: the Contemporary Use of Territorio in Latin American Geography. Journal of Latin American Geography, 16(1), 43-67.

» Lugones, M. (2010). Toward a Decolonial Feminsim. Hypatia, 25(4), 742-759.

》Manzano, V. (2007). Un barrio, diferentes grupos: Acerca de dinámicas políticas locales en el distrito de la Matanza. En A. Grimson, C.M.C. Ferraudi y R. Segura, La vida política en los barrios populares de Buenos Aires (pp. 267-294). Buenos Aires: Prometeo.

» Manzano, V. (2015). Lugar, trabajo y bienestar: la organización barrial Tupac Amaru en clave de política relacional. Publicar, 13(19), 9-35.

» Massetti, A., Villanueva, R. y Gómez, M. (Eds.). (2010). Movilizaciones, Protestas e Identidades Políticas en la Argentina del Bicentenario. Buenos Aires: Nueva Trilce.

》Massey, D. (2005). For Space. London: SAGE.

"Massidda, A. (2018). Utopian Visions for Buenos Aires Shantytowns: Collective Imaginaries of Housing Rights, Upgrading and Eviction (1956-2013). Bulletin of Latin American Research, 37(2), 144-159.

»Mazzeo, M. (2014).Piqueter@s: Breve historia de un movimiento popular argentino. Buenos Aires: Editorial Quadrata.

》 Mazzeo, M., Acha, O. y Canpione, D. (2007). Reflexiones sobre el poder popular. Buenos Aires: Editorial El Colectivo.

» Menazzi, L. (2008). Construyendo al barrio: la postulación del barrio como territorio político durante la transición democrática. Argumentos, 10, 1-24.

» Merklen, D. (2005). Pobres Ciudadanos: Las Clases Populares en la Era Democrática (Argentina 1983-2003). Buenos Aires: Editorial Gorla.

" Mignolo, W.D. (1995). The Darker Side of the Renaissance: Literacy, Territoriality, and Colonization. Michigan: University of Michigan Press.

» Mignolo, W.D. (2003). Historias locales/diseños globales: colonialidad, conocimientos subalternos y pensamiento fronterizo. Madrid: Akal.

»Moore, D.S. (2005). Suffering for Territory: Race, Place, and Power in Zimbabwe. Durham: Duke University Press.

》 Mora, M. (2015). The politics of Justice: Zapatista autonomy at the margins of the neoliberal mexican state. Latin American and Caribbean Ethnic Studies, 10(1), 87-106.

》 MTD Almirante Brown. (2002). Los Movimientos de Trabajadores Desocupados y la construcción del poder popular. Herramienta 21. Recuperado de: http:// www.herramienta.com.ar/print/revista-herramienta-n-21/los-movimientos-detrabajadores-desocupados-y-la-construccion-del-poder-pop

》 Muxica, L.S.M. (2011) La favela como espacio de exclusión social en la ciudad de Rio de Janeiro. IEURE, 37(110), 117-132.

»Natalucci, N., Pérez, G., Schuster, F. y Gattoni, M.S. (2013). Territorios disputados: Movilización política y procesos de institucionalización en niveles locales de gobierno (Argentina, 2003-2011). Revista Mexicana de Análisis Político y Administración Pública, 2(2), 139-159.

» Natalucci, A. y Pérez, G. (2015). La imaginación institucional. Movimientos sociales y Estado en Argentina (2003-2015). II Congreso FLACSO. 
» Naylor, L. (2017). Reframing autonomy in political geography: A feminist geopolitics of autonomous resistance. Political Geography, 58, 24-35.

» Nel·lo, O. (2003). Aquí no! Conflictes territorials a Catalunya. Barcelona: Empúries.

»Offen, K.H. (2003). The Territorial Turn. Journal of Latin American Geography, 2(1), 43-73.

» Oslender, U. (2016). The Geographies of Social Movements: Afro-Colombian Mobilization and the Aquatic Space. Durham: Duke University Press.

»Painter, J. (2010). Rethinking Territory. Antipode, 42(5), 1090-1118.

»Perlman, J.E. (1976). The myth of marginality. Urban poverty and politics in Rio de Janeiro. California: Univeristy of California Press.

»Pérez-Wilke, I. (2014). Los blocos afro en Bahía. Máquinas de re-creación del territorio negro. Revista Politeia, 52(37), 119-138.

»Pérez-Wilke, I. y Marquez, F. (Eds.). (2013). Nuestra America Negra: Territorios y voces de la interculturalidad afrodescendiente. Caracas: Universidad Bolivariana de Venezuela.

»Porto-Gonçalves, C.W. (2002). Da Geografia às Geo-grafias: um mundo em busca de novas territorialidades. En A.E. Ceceña (Ed.), A Guerra Infinita: hegemonia e terror mundial (pp. 359-391). Rio de Janeiro: CLACSO.

》 Porto-Gonçalves, C.W. (2012). A reinvenção dos territórios na América Latina/Abya Yala. Universidad Autónoma de México, http://www2.fct.unesp.br/docentes/geo/ raul/biogeografia_saude_publica/aulas\%202014/2-carlos\%20walter.pdf

»Quirós, J. (2008). Piqueteros y peronistas en la lucha del Gran Buenos Aires. Por una visión no instrumental de la política popular. Cuadernos de Antropología Social, 27, 113-131.

»Radcliffe, S.A. (2012). Development in a postneoliberal era? Sumak Kawsay, living well and the limits to decolonialisation in Ecuador. Geoforum, 43, 240-249.

» Raffestin, C. (1980). 2015. Por Una Geografia del Poder. Madrid: Traficantes

»Restrepo, P. y Valencia, J. (2016). Prácticas comunicativas en el Buen Vivir. En P. Restrepo, J. Valencia y C. Maldonado (Eds.), Comunicación, movilizaciones sociales y postdesarrollo: la revolución sí está sucediendo (pp. 35-60). Quito: Ciespal.

》 Retamozo, M., Schuttenberg, M. y Viguera, A. (Eds.). (2013). Peronismos, Izquierdas y Organizaciones Populares: Movimientos e identidades políticas en la Argentina contemporánea. La Plata: EDULP.

》 Reyes, A. y Kaufman, M. (2011). Sovereignty, Indigeneity, Territory: Zapatista Autonomy and the New Practices of Decolonialization. The South Atlantic Quarterly, 110(2), 505-525.

》 Rivera Cusicanqui, S. (2010). Ch'ixinakax utxiwa: Una reflexión sobre prácticas y discursos descolonizadores. Buenos Aires: Tinta Limón.

» Rivero, C.V. (2014). La descolonización del territorio: Territorialidad campesina y Estado multiterritorial abigarrado. Veredas, 28, 523-557.

» Rosell Arce, P. (2012). 2011: ¿El parteaguas del Evismo? Bolivia después del conflicto del Tipnis. Revista Nueva Sociedad, 237. Recuperado de: www.nuso.org 
» Rosset, P. (2013). Re-thinking agrarian reform, land and territory in La Via Campesina. The Journal of Peasant Studies, 40(4), 721-775.

» Rossi, F.M. (2015). Beyond Clientelism: The Piquetero Movement and the State in Argentina. En P. Almeida y A.C. Ulate (Eds.), Handbook of Social Movements across Latin America (pp. 117-128). New York: Springer.

» Rossi, F.M. (2017). The Poor's Struggle for Political Incorporation: The Piquetero Movement in Argentina. Cambridge: Cambridge University Press.

» Routledge, P. (2015). Territorialising movement: the politics of land occupation in Bangladesh. Transactions of the Institute for British Geographers, 40(4), 445-463.

»Sack, R. (1986). Human Territoriality: its theory and history. Cambridge: Cambridge University Press.

»Santos, M. (2000). La naturaleza del espacio. Técnica y tiempo. Razón y emoción. Barcelona: Editorial, Ariel Geografía.

» Satizabal, P. y Batterbury, S.P.J. (2018). Fluid geographies: Marine territorialisation and the scaling up of local aquatic epistemologies on the Pacific coast of Colombia Transactions of the Institute of British Geographers, 43(1), 61-78.

»Sesia, P. y Sarmiento, S. (Eds.). (2008). Pueblos indígenas, territorios y género en el México rural contemporáneo. Mexico City: AMER / Casa Juan Pablos / UAM / CON- ACyT / UAEM / Universidad Michoacana de San Nicola's de Hidalgo.

» Sharp, J.P., Routledge, P., Philo, C. y Paddison, R. (Eds.). (2000). Entanglements of Power. New York: Routledge.

» Silva, L.L. (2008). Breve relato histórico da luta por moradia em Salvador: 0 caso da ocupação Quilombo de Escada. Antíteses, 1(1), 118-150.

» Soja, E.W. (1971). The Political Organization of Space. Washington: Association of American Geographers.

» Soja, E.W. (1996). Thirdspace: Journeys to Los Angeles and Other Real-andImagined Places. Oxford: Blackwell.

»Souza, M.L. de (1995). O território: Sobre espaço e poder, autonomia e desenvolvimento. En I.E. de Castro, P.C. da Costa Gomes y R.L. Correa (Eds.), Geografia: Conceitos e temas (pp. 77-116). Rio de Janeiro: Bertrand Brasil.

»Souza, M.L. de (2008). Fobópole: O medo generalizado e a militarização da questão urbano. Rio de Janeiro: Bertrand Brasil.

»Souza, M.L. de (2015a). Dos Espaços de Controle aos Terriórios Dissidentes: Escritos de divulgação científica e análise política. Rio de Janeiro: Consequencia.

» Souza, M.L. de (2015b). Lessons from Praxis: Autonomy and Spatiality in Contemporary Latin American Social Movements. Antipode, 48(5), 1292-1316.

» Steinberg, P.E. (2009). Sovereignty, Territory, and the Mapping of Mobility: A View from Outside. Annals of the Associations of American Geographers, 99(3), 467495.

» Storey, D. (2012). Territories: The Claiming of Space. London: Routledge.

»Stratta, F. y Barrera, M. (2009). El tizón encendido: protesta social, conflicto y territorio en la Argentina de la postdictadura. Buenos Aires: Editorial El Colectivo.

»Svampa, M. y Pereyra, S. (2003). Entre la ruta y el barrio: La experiencia de las 
organizaciones piqueteras. Editorial Biblios.

"Svampa, M. (2015). Commodities Consensus: Neoextractivism and enclosure of the commons in Latin America. South Atlantic Quarterly, 114(1), 65-82.

»Svampa, M. (2017). Del Cambio de Época al Fin del Ciclo: Gobiernos progresistas, extractivismo y movimientos sociales en América Latina. Buenos Aires: Edhasa.

"Swyngedouw, E. (2004). Globalisation or 'glocalisation'? Networks, territories and rescaling. Cambridge review of international affairs, 17(1), 25-48.

» Tapia, L. (2002). La condición multisocietal: multiculturalidad, pluralismo, modernidad. La Paz: Muela del Diablo Ed.

» Taylor, P.J. (1994). The state as container: territoriality in the modern worldsystem. Progress in Human Geography, 18(2), 151-162.

» Tobío, O. (2012). Territorios de la incertidumbre: Apuntes para una geografía social. Buenos Aires: UNSAM.

» Torres, F. (2011). Territorio y lugar: potencialidades para el análisis de la constitución de sujetos políticos. El caso de un movimiento de desocupados en Argentina. Geograficando, 7(7), 209-238.

"Ulloa, A. (2011). The Politics of Autonomy of Indigenous Peoples of the Sierra Nevada de Santa Marta, Colombia: A Process of Relational Indigenous Autonomy. Latin American and Caribbean Ethnic Studies, 6(1), 79-107.

》 Ulloa, A. (2012). Los territorios indígenas en Colombia: de escenarios de apropiación transnacional a territorialidades alternatives. Scripta Nova, 16. Recuperado de: http://www.ub.edu/geocrit/sn/sn-418/sn-418-65.htm

"Varea, S. y Zaragocin. S. (Eds.). (2017). Feminismo y Buen Vivir: Utopias Decoloniales. Cuenca: Pydlos.

» Varley, A. (2013). Postcolonialising informality? Environment and Planning D: Society and Space, 31, 4-22.

»Varley, A. (2017). Property titles and the urban poor: from informality to displacement? Planning Theory and Practice, 18(3), 385-404.

»Wahren, J. (2011). Territorios Insurgentes: La dimensión territorial en los movimientos sociales de América Latina. IX Jornadas de Sociología. Facultad de Ciencias Sociales, Universidad de Buenos Aires: Buenos Aires.

"Wainwright, J. (2008). Decolonizing Development: Colonial Power and the Maya. Oxford: Blackwell Publishing.

»Wainwright, J. y Bryan, J. (2009). Cartography, territory, property: postcolonial reflections on indigenous counter-mapping in Nicaragua and Belize. Cultural geographies, 16(2), 153-178.

»Walsh, C. (2010). Development as buen vivir: institutional arrangements and (de) colonial entanglements. Development, 53(1), 15-21.

》Zibechi, R. (2007). Autonomías y emancipaciones América Latina en movimiento. Fondo Editorial de la Facultad de Ciencias Sociales: Unidad de Post Grado: UNMSM

"Zibechi, R. (2012). Territories in Resistance: A Cartography of Latin American Social Movements. Oakland: AK Press. 
SAM HALVORSEN

»Zuñiga Navarro, G. (1998). Los procesos de constitución de territorios indígenas en América Latina. Nueva Sociedad, 153, 141-155.

Dr. Sam Halvorsen / s.halvorsen@qmul.ac.uk Licenciado en Geografia (University of Bristol), Master en Geografía (University College London) y Doctor en Geografía (Univeristy College London). Docente e Investigador (Queen Mary University of London). Publicó artículos en revistas geográficas como Transactions of the Institute of British Geographers y Annals of the American Association of Geographers. 